\title{
LA MÉMOIRE DE NOS MAîTRES : LA COOPÉRATION ENTRE LES HISTORIENS DE POZNAŃ ET DE TORUŃ
}

Stanisław ROSZAK

Dans cet exposé sur le rôle du professeur Jerzy Topolski en tant qu'historien, je voudrais me concentrer sur les questions que je connais le mieux et qui me sont les plus proches. Il s'agit de la coopération scientifique entre les milieux historiques de Poznań et de Toruń, en particulier dans le domaine de l'époque moderne et du XVIII ${ }^{e}$ siècle. À ce sujet, il faut distinguer, en plus du professeur Jerzy Topolski, trois personnalités centrales : pour Poznań, le professeur Maciej Serwański et le professeur Marian Drozdowski et, du côté de Toruń, le professeur Jacek Staszewski. Il convient de souligner ici l'influence de l'école de méthodologie historique de Poznań (notamment avec le professeur Wojciech Wrzosek qui pendant une courte période de travail à Torun a fait école - je ne mentionnerai ici que deux de ses élèves : le professeur Wojciech Piasek et le docteur Tomasz Falkowski qui a déménagé de Toruń à Poznań).

Mon témoignage se concentrera sur trois événements qui, à mon avis, ont eu un grand impact sur l'établissement des relations particulières entre l'Université Nicolas Copernic et l'Université Adam Mickiewicz, ainsi que sur les orientations des recherches scientifiques et les liens d'amitié qui durent jusqu'à aujourd'hui. 


\section{POZNAŃ 1988 - UN NOUVEAU REGARD SUR L'HISTOIRE DU XVIIle SIĖCLE}

Le premier événement, c'est la conférence consacrée aux relations politiques, économiques et culturelles de la Pologne et de la Saxe, organisée à Poznań en 1988 par Antoni Czubiński, Jerzy Topolski et Marian Drozdowski'. Au cours des quatre journées de discussions, on a présenté quarante exposés qui ont non seulement apporté la connaissance de nombreux nouveaux faits historiques mais ont aussi changé la perception de la période du règne des Saxons et de la Maison des Wettin dans l'histoire et l'historiographie polonaise. A l'époque du règne de Stanisław August Poniatowski, cette période - correspondant à la première moitié du XVIII siècle était considérée aussi bien dans l'historiographie polonaise qu'allemande comme un âge sombre, une période d'échecs politique, économique et culturel. Selon cette perception, ce n'est que dans la seconde moitié du XVIII ${ }^{\mathrm{e}}$ siècle, avec l'avènement de Poniatowski, qu'auraient été apportés des changements et des réformes positifs, associés aux débuts de l'époque des Lumières. La conférence de Poznań a changé cette vision simplifiée du XVIII ${ }^{\text {e }}$ siècle en Pologne et a jalonné de nouvelles orientations de recherches, ce qui a été souligné, du côté polonais, par le professeur Staszewski (Université Nicolas Copernic de Toruń) et, du côté allemand, par le professeur Klaus Zernack (Freie Universität Berlin). La conférence a été l'occasion de confronter aussi bien différentes écoles de recherche que différentes générations de chercheurs. Y sont intervenus de jeunes historiens, aujourd'hui des professeurs estimables - Wojciech Kriegseisen (aujourd'hui directeur de l'Institut d'Histoire de l'Académie Polonaise des Sciences à Varsovie), Krzysztof Mikulski (aujourd'hui président de la Société Historique Polonaise), Bogusław Dybaś (aujourd'hui directeur du Centre de recherche scientifique de l’Académie Polonaise des Sciences à Vienne).

Cette conférence a par ailleurs confirmé la position des divers centres scientifiques polonais travaillant sur le $\mathrm{XVIII}^{\mathrm{e}}$ siècle - position qui rappelait un peu la distribution du pouvoir sur la carte politique du XVIII ${ }^{\mathrm{e}}$ siècle. Nous pouvons comparer cet ensemble au concert européen des cinq grandes puissances du XVIII ${ }^{\mathrm{e}}$ siècle - la France, l’Angleterre, l'Autriche, la Prusse et la Russie. Dans la Pologne des années 80 . et 90 , dominaient en effet cinq milieux scientifiques dixhuitièmistes Cracovie (les chercheurs regroupés autour de Józef Andrzej Gierowski), Varsovie (autour de Jerzy Michalski), Toruń (autour de Jacek Staszewski et Jerzy Wojtowicz),

M. Drozdowski, K. Maliszewski, Konferencja w Poznaniu o związkach Polski i Saksonii w czasach nowożytnych, „Roczniki Dziejów Społecznych i Gospodarczych”, t. 49, 1988, p. 285-287. 
Gdańsk (autour de Edmund Cieślak) et Poznań (l'histoire socio-économique de Jerzy Topolski et l'histoire du régime politique de Henryk Olszewski).

Les contributions de cette conférence n'ont pas été publiées dans l'immédiat. Un an après, nous assistions à la chute du communisme. Par conséquent, les historiens avaient d'autres préoccupations. Ce n'est que cinq ans plus tard, après plusieurs résolutions importantes prononcées à Poznań, qu'elles ont été publiées. À l'occasion du soixantième anniversaire du professeur Jacek Staszewski, a été publié à Toruń le livre Entre la grande politique et la province nobiliaire. Etudes sur l'histoire de la Pologne et de $l^{\prime}$ Europe moderne $e^{2}$. Le livre commençait par deux articles des collègues de Poznań celui de Henryk Olszewski « L'époque des Saxons dans l'opinion de l'historiographie polonaise » et celui de Jerzy Topolski, « L'époque des Saxons a-t-elle existé en Pologne ? Sur les implications méthodologiques de la périodisation historique ».

Jerzy Topolski a exprimé un point de vue audacieux sur la division de l'époque du règne des Saxons. En se servant des conclusions émises par les historiens du régime politique, de l'économie et de la culture, il a souligné les dissemblances entre les règnes des deux rois Wettin. Nous donnons la parole à l'auteur : « J'arrive ici au point le plus important de mon essai - écrivait Topolski - Il s'agit d'un changement général de point de vue sur l'époque saxonne. Cependant, dans la périodisation de l'histoire polonaise, cela exige de séparer la période du règne d'Auguste II de celle du règne d'Auguste III. En faisant une telle déconstruction de l'époque saxonne, ce qui me semble indispensable, il faudrait attacher l'époque du règne d'Auguste II à l'époque précédente tandis que celle du règne d'Auguste III à l'époque suivante, donc à l'époque du règne de Stanisław August Poniatowski. Cela signifie que nous situons l'un des plus importants tournants de l'histoire polonaise en 1733, et non plus comme d'habitude en $1764 \gg^{3}$.

De cette façon, l'historien introduisait l'époque d'Auguste III dans le siècle des Lumières. Il se servait de nouveaux arguments du domaine de l'histoire économique, en analysant notamment le territoire de la Grande-Pologne (il mettait en reliefle développement économique de la Grande-Pologne ainsi que celui de Poznań ; il signalait la croissance du commerce avec la Saxe, l'afflux de nouveaux colons à la campagne et en ville, la croissance de l'élevage, du nombre de moutons). En tant qu'historien

2 Między wielka polityką a szlacheckim partykularzem. Studia z dziejów nowożytnej Polski i Europy, sous la direction de Kazimierz Wajda, Toruń, 1993.

3 Jerzy Topolski, Czy istniała w Polsce epoka saska? Czyli metodologiczne implikacje periodyzacji historycznej, dans Między wielka polityką a szlacheckim partykularzem, op. cit., p. 39: „I tu docieram do punktu w tym szkicu dla mnie najważniejszego. Chodzi o ogólną zmianę punktu widzenia na czasy saskie. To jednak wymaga rozdzielenia w periodyzacji historii Polski czasów Augusta II i czasów Augusta III. Dokonując takiej niezbędnej mym zdaniem destrukcji epoki saskiej należałoby czasy Augusta II związać z epoką poprzednią, natomiast czasy Augusta III z epoką następną, czyli czasami stanisławowskimi. Oznacza to postawienie jednej z zasadniczych cezur w polskiej historii na roku 1733, a nie jak to zwykle bywa - na roku 1764". 
économique il constatait : «De toute façon, l'historien économique ne peut être d'accord avec le point de vue selon lequel les changements positifs dans l'économie polonaise du XVIII siècle n'ont commencé qu'après 1764, donc avec les débuts du règne du roi Stanisław August Poniatowski $\gg^{4}$.

Dans ses travaux postérieurs, en particulier dans ses synthèses de l'histoire polonaise ${ }^{5}$ et dans son manuel d'histoire pour l'école primaire ${ }^{6}$, Topolski a utilisé cette périodisation nouvelle (il était un des rares historiens et méthodologues qui essayait d'introduire seul ses propres résolutions scientifiques dans le système d'éducation) ${ }^{7}$.

\section{L'ANNÉE 1996 - SÉMINAIRE SCIENTIFIQUE À OBRZYCKO}

Le deuxième événement, c'est le séminaire organisé par Jerzy Topolski et Jacek Staszewski à Obrzycko, près de Poznań dans un centre de conférences de l'Université Adam Mickiewicz. C'était l'année 1996. Ce séminaire a regroupé des méthodologues d'histoire et des historiens de l'époque moderne, des collaborateurs et des élèves du professeur Topolski. Le débat concernait, entre autres, les problèmes liés à l'éducation à l'époque des Lumières ainsi qu'aux diètes et diétines du XVIII siècle (je me souviens aussi d'une thématique spécifique concernant les cabinets de physique au XVIII ${ }^{\mathrm{e}}$ siècle). Après toutes ces années, ce qui me semble aujourd'hui le plus important, c'est le fait que les jeunes chercheurs de Toruń et de Poznań ont pu alors se rencontrer et entamer des discussions, sous le patronage des maîtres célèbres. Parmi les jeunes chercheurs et étudiants de Poznań, il y avait Maciej Forycki et Michał Zwierzykowski, aujourd'hui professeurs de l'Université Adam Mickiewicz.

Le professeur Marian Drozdowski a joué un rôle inestimable dans l'intégration du milieu. Je me rappelle encore aujourd'hui quand le professeur Drozdowski, en se souciant que le dîner soit bien préparé, a demandé au professeur Topolski de raccourcir la discussion en faisant référence à la conception méthodologique de Frank Ankersmit. Je cite de mémoire : « La nuit tombe, le dîner est fait - Ankersmit et sa conception peut attendre tandis que l'échine grillée n'attendra pas $\gg$. Les professeurs Topolski et Staszewski ont tous deux approuvé la justesse de cette proposition.

4 Ibidem, p. 40: „Historyk gospodarczy w każdym razie nie może zgodzić się z poglądem, że pozytywne zmiany w gospodarce polskiej XVIII wieku zaczęly się dopiero po 1764 czyli z początkiem panowania króla Stanisława Augusta Poniatowskiego”.

5 Cf. idem, Polska w czasach nowożytnych. Od środkowoeuropejskiej potęgi do utraty niepodlegtości (15011795), Poznań, 1994.

6 Idem, Trudny lot orła biatego. Rozmowy o historii, 1997; idem, Uczymy się historii. Klasa 4 szkota podstawowa, Polska Oficyna Wydawnicza BGW 1995.

7 Au sujet de la perception de la méthodologie dans les manuels scolaires voir: V. Julkowska, Dlaczego orzeł a nie lew? Dialog Jerzego Topolskiego z dziećmi o historii, „Przegląd Bydgoski”, 11: 2000, p. 45 -56 et aussi; eadem, Zmiana modelu metodologicznego podręczników historii, „Sensus Historiae”, 2013: 5, p. 71-81. 


\section{L'ANNÉE 2004 - CONFÉRENCE SCIENTIFIQUE À TORUŃ}

Enfin le troisième événement à mon avis essentiel pour la coopération, c'est la conférence : Noblesse française et noblesse polonaise : Mémoire, identité, culture, $X V I^{e}$ $X X^{e}$ siècles, organisée à Toruń en 2004, grâce à la coopération du professeur Jarosław Dumanowski et du professeur Michel Figeac. En tant que participant, je peux attester que les collègues modernistes de Poznań - surtout l'excellent expert des relations franco-polonaises, le professeur Maciej Serwański, ainsi que ses plus jeunes collègues, Maciej Forycki, Igor Kraszewski et Agnieszka Jakuboszczak, aujourd'hui chercheurs connus de l'histoire du XVII ${ }^{\mathrm{e}}$ et XVIII ${ }^{\mathrm{e}}$ siècles - ont joué un rôle significatif dans la conférence elle-même ainsi que dans l'établissement de contacts fructueux avec le centre scientifique en France.

À la fin de mon propos commémoratif, je voudrais souligner le rôle important des écoles historiques et des centres scientifiques dans le développement des recherches. Je suis certain que dans le cas du professeur Jerzy Topolski et du professeur Jacek Staszewski, on peut parler de leur rôle fondateur dans la coopération entre Poznań et Toruń. Grâce aux articles et livres de leurs collaborateurs, élèves et successeurs, la coopération dure jusqu'à aujourd'hui. La mémoire de nos Maîtres scientifiques est un élément important de la durabilité de cette coopération. 